\title{
Meta
}

Journal des traducteurs

Translators' Journal

Pragana Dantas, Marta et Henriques Pereira de Souza, Germana (dir.) (2016) : A tradução de obras francesas no Brasil : trajetórias, debates, deslocamentos. Campinas : Pontes Editores, $184 \mathrm{p}$.

\section{Denise Capra}

Volume 63, numéro 1, avril 2018

URI : https://id.erudit.org/iderudit/1050528ar

DOI : https://doi.org/10.7202/1050528ar

Aller au sommaire du numéro

Éditeur(s)

Les Presses de l’Université de Montréal

ISSN

0026-0452 (imprimé)

1492-1421 (numérique)

Découvrir la revue

Citer ce compte rendu

Capra, D. (2018). Compte rendu de [PRAGANA DANTAS, Marta et HenriQues PEREIRA DE SOUZA, Germana (dir.) (2016) : A tradução de obras francesas no Brasil : trajetórias, debates, deslocamentos. Campinas : Pontes Editores, 184 p.] Meta, 63(1), 263-264. https://doi.org/10.7202/1050528ar d'utilisation que vous pouvez consulter en ligne. 
5. Par exemple, Jean Vinay est écrit par erreur au lieu de Jean Darbelnet (p. 57, ligne 5); translation au lieu de translators (p. 62, avant-dernier paragraphe); on voit la répétition du contenu des pages 74 à 87 dans les pages 96 à 109; de nombreuses fautes de frappe (p. 129, 132, 144, $145,146,154,173,174,409)$, pour ne citer que celles-là. Nous espérons que l'auteur aura l'occasion de les corriger dans une prochaine édition.

\section{RÉFÉRENCES}

Asobele, Timothy (1999): New Perspectives in the Training of Translators and Interpreters in Nigeria. Lagos: Printview Publishers.

Bandia, Paul (2005): Esquisse d'une histoire de la traduction en Afrique. Meta. 50(3):957-971.

Bandia, Paul (2001): African Tradition. In: Mona BAKER (dir.). Routledge Encyclopedia of Translation Studies. New York: Routledge, 295-305.

Simpson, Ekundayo (2007a): Translation Interventions: Papers and Addresses on Translation, Language, Literature and Culture. Lagos/ Abuja: Interlingua Limited.

Simpson, Ekundayo (2007b): Translation: Principles and Applications. Lagos/Abuja: Interlingua Limited.

Simpson, Ekundayo (1985): Translation Problems of African Countries. In: Hildegund BüHLER (dir.). Translators and Their Position in Society. Proceedings of the 10th World Congress of FIT. Vienne: Braumüller, 105-110.

Pragana Dantas, Marta et Henriques Pereira De SouzA, Germana (dir.) (2016): A tradução de obras francesas no Brasil: trajetórias, debates, deslocamentos. Campinas: Pontes Editores, $184 \mathrm{p}$.

Ce volume du recueil Histórias da Tradução regroupe les interventions survenues au cours de la III ${ }^{e}$ Rencontre des traducteurs d'œuvres littéraires françaises au Brésil, organisée conjointement par le Programme de spécialisation en lettres et la Coordination du programme de baccalauréat en traduction de l'Université fédérale de la Paraíba, qui a eu lieu en octobre 2014 à João Pessoa. Ce livre est la suite des recueils précédents organisés à l'occasion des $\mathrm{I}^{\text {re }}$ et $\mathrm{II}^{\mathrm{e}}$ Rencontres des traducteurs d'œuvres littéraires françaises, qui ont eu lieu respectivement en 2006 et 2009 . Il ressort des articles qui composent ce recueil que les littératures nationales, par le biais de la traduction, entrent en compétition avec d'autres traductions et modèles, de manière directe ou implicite, dans l'analyse des œuvres françaises traduites au Brésil.

Le recueil débute par une réflexion à propos des inégalités et des asymétries qui régissent la circulation internationale de la littérature. Dans Éléments pour une cartographie de la littérature québécoise au Brésil: textes, parcours, acteurs ${ }^{1}$, Marc Charron se penche sur deux romans québécois, Une histoire américaine ${ }^{2}$ de Jacques Godbout et $R u^{3}$ de Kim Thúy. L'auteur remet en question le rôle centralisateur de la France dans la circulation des littératures en français, y compris dans la littérature québécoise, en abordant la pertinence de faire coïncider la discussion sur la traduction dans l'espace latino-américain et la problématisation de l'américanité partagée.

En revanche, dans Transpiration et récréation poétiques: une réflexion critique sur la traduction brésilienne d' "Exercice de style» de Raymond Que$n e a u^{4}$, Lia Araújo, M. de Lima et Germana Henriques Pereira de Sousa examinent les choix faits par Luiz Rezende dans la traduction brésilienne d'Exercices de style ${ }^{5}$ de Queneau. Les auteures, évoquant l'éthique dans le processus de la traduction, invitent les lecteurs à des questionnements théoriques sur les choix du traducteur quant à la visibilité de l'Autre et la traduction ethnocentrique.

Marie-Hélène C. Torres établit, dans Le pouvoir anthropophagique du traducteur: entre tradition et innovation ${ }^{6}$, un dialogue avec plusieurs théoriciens de la traduction. Elle problématise la figure du traducteur comme anthropophage, celui qui révèle ou annexe l'étranger, et met en regard les stratégies de traduction et la question de l'invisibilité. L'auteure présente deux questions fondamentales: l'agentivité du traducteur à travers ses stratégies et la lutte consciente, ou non, pour la visibilité ou la reconnaissance internationale d'une littérature.

Elle-même traductrice, Ivone C. Benedetti présente dans Traduction des sciences humaines: quelques questions à propos de la formation des traducteurs $^{7}$ une réflexion sur la préparation du traducteur au marché du travail. En mettant l'accent sur la formation dans les humanités françaises au Brésil, le texte reprend l'historique des transformations survenues au cours des cinquante dernières années dans le système d'éducation brésilien. La philosophie a été éliminée du cursus et l'histoire a perdu son statut de matière critique. Benedetti analyse les conséquences de ces lacunes pour la formation du futur traducteur qui travaille dans le domaine des sciences humaines.

Dans Les réserves de la traductrice: traduire d'autres domaines avec la psychanalyse ${ }^{8}$, Márcia Atalla Pietroluongo narre l'expérience d'une traductrice officielle au Brésil devant traduire le verbe réserver, dans le cadre d'une décision de justice en français. À partir de son histoire, il est possible de suivre les hésitations, les angoisses et les prises de décision qu'implique la traduction, surtout dans un texte juridique ayant un impact réel sur 
les personnes impliquées dans le procès. Les six parties prenantes de l'histoire créent une trame dont l'aboutissement permet à l'auteure de montrer de quelle manière la psychanalyse peut aider à mieux comprendre la subjectivité du traducteur dans le texte traduit.

Teresa Dias Carneiro, dans Préfaces de traducteurs d'ouvres littéraires françaises traduites vers le portugais au Brésil à partir de la moitié du $X X^{e}$ : une occasion ratée ?', souligne l'importante contribution à l'étude du paratexte du livre traduit au Brésil. L'auteure analyse 102 préfaces de traducteurs dans 360 exemplaires de traductions. Il ressort de son analyse que, dans la plupart des cas, les préfaces des traducteurs sont utilisées à des fins didactique et informative, puisque l'on y mentionne l'auteur et l'école littéraire à laquelle il est associé. Même dans les cas où il est question de la traduction dans la préface, il n'y a pas de discussion sur les implications ou les choix d'un traducteur. Dans son texte, Carneiro présente un parcours clair et simple qui systématise les aspects qui seront observés dans l'étude du paratexte du livre traduit.

Dans Traduire la comtesse: variations sur la violence dans Mémoires d'un âne, de la Comtesse de Ségur ${ }^{10}$, Vera Chacham examine la trajectoire de l'auteure d'origine russe en France, puis la traduction/adaptation de ses œuvres au Brésil. Chacham examine les aspects structurels de l'œuvre littéraire, tels que la notion d'éducation au XIX ${ }^{\mathrm{e}}$ siècle, surtout en ce qui a trait à la violence. L'auteure y inclut des informations sur le processus d'acclimatation de Mémoires d'un âne ${ }^{11}$ au Brésil réalisé par le traducteur Herberto Sales.

Dans Alector, histoire fabuleuse: questions sur la ponctuation et le sens ${ }^{12}$, Ivone Greis analyse l'ouvrage ${ }^{13}$ de Barthélemy Aneau, publiée en France en 1560 par Pierre Fradin. Il s'agit d'une analyse sur la relation complexe entre la ponctuation du texte original et celle choisie par Greis en portugais. L'objectif de l'auteure est de vérifier la construction du sens résultant du processus de transfert.

Le dernier article du livre, Anthologie brésilienne de Guy de Maupassant: paratextes ${ }^{14}$ traite de la présentation que Carmen Verônica de Almeida Ribeiro Nóbrega et Marie-Hélène Catherine Torres font de la vie et de l'œuvre de Maupassant et de la réception d'une partie de son œuvre au Brésil par le biais de paratextes insérés dans l'anthologie en question. Les auteures considèrent les paratextes comme une carte de présentation qui jette un pont entre l'auteur, le traducteur et le lecteur. Ainsi, les deux auteures évoquent les questions de la réception, des politiques éditoriales et du rapport avec le système national.

Regroupant un large éventail de travaux dans le domaine des études de la traduction, le livre souligne la relation dynamique et productive de l'importation et de l'exportation de littérature en langue française au Brésil, de la France du $\mathrm{XVI}^{\mathrm{e}}$ siècle à la littérature québécoise.

\section{Denise CAPra \\ Université de Montréal, Montréal, Canada}

\section{NOTES}

1. Elementos para uma cartografia da literatura quebequense traduzida no Brasil: textos, percursos, atores.

2. Godbout, Jacques (1986): Une histoire américaine. Paris: Seuil.

3. Thúy, Kim (2009): Ru. Montréal: Libre Expression.

4. Transpiração e recreação poéticas: uma reflexão crítica sobre a tradução brasileira de Exercices de style, de Raymond Queneau.

5. Queneau, Raymond (1947): Exercices de style. Paris: Gallimard.

6. O poder antropofágico do tradutor: entre tradição e inovação.

7. Tradução de ciências humanas: algumas questões em torno da formação dos tradutores.

8. As reservas da tradutora: traduzindo outros campos com a psicanálise.

9. Prefácios de tradutores em obras francesas traduzidas para o português no Brasil a partir de meados do século XX: uma oportunidade perdida?

10. Traduzindo a condessa: variações sobre a vilência em Memórias de um burro, da Condessa de Ségur.

11. Rostopchine, Sophie (comtesse de Ségur) (1860): Mémoires d'un âne. Paris: Hachette.

12. Alector, histoire fabuleuse: questões sobre pontuação e sentido.

13. Aneau, Barthélemy (1560): Alector ou le Coq. Lyons: Pierre Fradin.

14. Antologia brasileira de Guy de Maupassant: paratextos.

Traduction et interprétation face aux défis actuels (2016): Revue Française de Linguistique Appliquée. XXI(1).

Après deux numéros consacrés à la traduction (Vol. VII-2, 2003 et Vol. XIV-1, 2009, accessibles en ligne à partir de rfla-journal.org), la Revue Française de Linguistique Appliquée fait à nouveau le point sur l'évolution de la recherche dans ce domaine et rassemble, dans sa livraison de juin 2016, sept articles reflétant la diversité et le dynamisme des études actuelles dans le champ de la traductologie, englobant traduction et interprétation.

Partant de l'observation que ce champ de pratiques et de recherches théoriques se trouve en pleine mutation, du double fait d'une nouvelle 